Article

\title{
On the Banzhaf-like Value for Cooperative Games with Interval Payoffs
}

\author{
Chunqiao Tan ${ }^{1} \mathbb{D}$, Wenrui Feng ${ }^{1}$ and Weibin Han $^{2, *}$ \\ 1 School of Business, Central South University, Yuelu District, Changsha 410083, China; \\ chunqiaot@sina.com (C.T.); wenruif@sina.cn (W.F.) \\ 2 School of Economics and Management, South China Normal University, Guangzhou Higher Education \\ Mega Center, No. 378, Waihuan Xi Road, Guangzhou 510006, China \\ * Correspondence: weibinhan@m.scnu.edu.cn
}

Received: 7 February 2020; Accepted: 2 March 2020; Published: 7 March 2020

\begin{abstract}
By using Moore's subtraction operator and a total order on the set of closed intervals, we introduce a new variation of the Banzhaf value for cooperative interval games called the interval Banzhaf-like value which may accommodate the shortcomings of the interval Banzhaf value. We first reveal the relation between this introduced value and the interval Banzhaf value. Then, we present two sets of properties that may be used to determine whether an interval value is median-indifferent to the interval Banzhaf-like value. Finally, in order to overcome the disadvantages of the interval Banzhaf-like value, we propose the contracted interval Banzhaf-like value and give an axiomatization of this proposed value.
\end{abstract}

Keywords: cooperative interval game; interval Banzhaf value; interval Banzhaf-like value; contracted interval Banzhaf-like value

\section{Introduction}

A cooperative game is represented by a pair $(N, v)$, where $N$ is a nonempty and finite set of players, and $v$ is a characteristic function from $2^{N}$ to $\mathbb{R}$ which assigns each coalition a real number. To cover a wider range of decision circumstances, the model of cooperative games was generalized either by changing the structure of the coalitions (See [1-3]), or by altering the nature of the characteristic function (See [4-6]). In the present paper, we study the model of cooperative interval games where the values of coalitions are assumed to be bounded and closed intervals of real numbers without any knowledge of related probability distribution on these obtained intervals.

The model of cooperative interval games was originally introduced by [7] to handle bankruptcy situations where the estate is known with certainty while claims belong to known bounded intervals of real numbers. Since then, it has been theoretically developed by a group of researchers around Branzei and Tijs (See $[4,8]$ for details). This model can be used to approach the situations where the players wish to cooperate and know with certainty only the lower and upper bounds for the payoff of their cooperation. Examples are bankruptcy problems in [7], minimum spanning tree networks in $[9,10]$, sealed bid second price auctions and flow situations in [8], etc.

The main focus of cooperative interval games are solutions that assign each given cooperative interval game a set of $n$-dimensional vectors which inform the players about the range of individual payoffs generated by cooperation. By using the arithmetic of intervals introduced by [11], the interval Shapley value and the interval Banzhaf value were separately proposed by $[12,13]$ for a class of cooperative interval games so called the size monotonic interval games. Moreover, these two values were separately characterized by using a set of properties. One common disadvantage of these two notions is that both are only well-defined for size monotonic interval games. Therefore, the axiomatization 
and properties concerning these values could only be provided for this specific subclass of cooperative interval games as well. To avoid this disadvantage of the interval Shapley value, the interval Shapley-like value was proposed in [14] by using Moore's subtraction operator. It has been shown that the interval Shapley-like value is definable for every possible cooperative interval games, and be characterized on the whole class of cooperative interval games.

Here, we introduce the notion of the interval Banzhaf-like value by using the interval subtraction operator in [11] so as to accommodate the mentioned disadvantage of the interval Banzhaf value introduced by [13]. It will be showed that the interval Banzhaf-like value is not only well-defined for every possible interval game, but also is median-indifferent to the interval Banzhaf value on the class of size monotonic interval games. Moreover, we offer two sets of properties about interval values. By using these properties, we may obtain the interval values that are median-indifferent to the interval Banzhaf-like value. Besides, as the interval Banzhaf-like value is provided with neither 2-players merging equivalence nor the uncertainty-removing property, we improve this value by introducing the contracted interval Banzhaf-like value, and present an axiomatization for this introduced value. It will be proved that the contracted interval Banzhaf-like value satisfies more desirable properties.

The rest of this paper is organized as follows. In Section 2, we recall the basic notions and operations with respect to the intervals of real numbers and the model of cooperative interval games. Section 3 is devoted to revealing the relation between the interval Banzhaf value and the interval Banzhaf-like value. In Section 4, we first show the properties of the interval Banzhaf-like value, and then present two sets of constraints to judge whether an interval value is median-indifferent to the interval Banzhaf-like value. Section 5 is dedicated to the contracted interval Banzhaf-like value and its axiomatization. We end this paper with a concluding remark in Section 6.

\section{Preliminaries}

This section is dedicated to the necessary notations, concepts and results concerning the intervals of real numbers and the model of cooperative interval games which are mainly from [11,14].

Let $\mathbb{R}$ be the real number set. Denote the set of all closed and bounded intervals on $\mathbb{R}$ by $I(\mathbb{R})$, and the length of interval $I \in I(\mathbb{R})$ by $|I|$. For any $\alpha \in \mathbb{R}$ and $I, J \in I(\mathbb{R})$ with $I=[\underline{I}, \bar{I}]$ and $J=[\underline{J}, \bar{J}]$, define that

- $\quad I \oplus J=[\underline{I}+\underline{J}, \bar{I}+\bar{J}]$

- $\quad I \ominus J=[\underline{I}-\bar{J}, \bar{I}-J] ;$

- $\alpha I=\left\{\begin{array}{lll}{[\alpha \underline{I}, \alpha \bar{I}]} & \text { if } \quad \alpha \geq 0 ; \\ {[\alpha \bar{I}, \alpha \underline{I}]} & \text { if } \quad \alpha<0 .\end{array}\right.$

These interval operators were originally introduced by [11], and frequently used to process interval calculations in the literature.

An alternative interval subtraction operator was proposed in [12] to approach the cooperative interval games as follows. For any $I, J \in I(\mathbb{R})$ with $I=[\underline{I}, \bar{I}]$ and $J=[\underline{J}, \bar{J}], I-J=[\underline{I}-\underline{J}, \bar{I}-$ $\bar{J}]$ if $\bar{I}-\bar{J} \geq \underline{I}-J$. This operator is only well-defined for an ordered interval pair $(I, J) \in D$ where $D=\{(I, J) \in I \bar{I}(\bar{R}) \times I(\mathbb{R}) \mid \bar{I}-\bar{J} \geq \underline{I}-J\}$, while the former subtraction operator is definable for every pair of closed intervals. Note that for any intervals $I, J \in I(\mathbb{R})$, if $I-J$ is well defined, then $I-J$ is a subset of $I \ominus J$.

Within the model of cooperative interval games, it is usually assumed that the payoffs of coalitions are bound closed intervals of real numbers without any knowledge of related probability distribution of these payoffs. That means, if $x \in[a, b]$ and $y \in[c, d]$, it is possible that $x-y \in[a-d, b-c]$ but $x-y \notin$ $[a-c, b-d]$ (given $b-d \geq a-c$ ). In view of this fact, it is more reasonable to employ the subtraction operator defined by [11] when dealing with the cooperative interval games in our framework.

A total order over the intervals was proposed by [14] in order to deal with wider range of interval comparisons. Let $I, J \in I(\mathbb{R})$ with $I=[\underline{I}, \bar{I}]$ and $J=[\underline{J}, \bar{J}]$. Suppose that

- $\quad I$ is median-superior to $J$, denoted by $I \succ J$, if $\frac{I+\bar{I}}{2}>\frac{I+\bar{J}}{2}$; 
- $\quad I$ is weakly median-superior to $J$, denoted by $I \succsim J$, if $\frac{I+\bar{I}}{2} \geq \frac{J+\bar{J}}{2}$;

- $\quad I$ and $J$ are median-indifferent to each other, denoted by $I \sim J$, if $\frac{I+\bar{J}}{2}=\frac{I+\bar{I}}{2}$.

As the above total order on the set of closed intervals is based on the medians of intervals (or the expectation of the uniform distribution), we rename the original terms in ([14] p. 463) so as to reflect this point.

A cooperative interval game is presented by a pair $(N, v)$ where $N$ is a nonempty finite set of players and $v: 2^{N} \rightarrow I(\mathbb{R})$ is the characteristic function with $v(\varnothing)=[0,0]$. For any $S \in 2^{N}$, $v(S)=[v(S), \overline{v(S)}]$ is the worth of coalition $S$ in $(N, v)$ where $v(S)$ and $\overline{v(S)}$ are respectively the minimal worth and the maximal worth that coalition $S$ could receive when players in coalition $S$ work together. For simplicity, denote $(N, v)$ by $v$ when $N$ is known from the context. Let $|S|$ be the cardinality of $S$ where $S \in 2^{N}$ and $I G^{N}$ be the family of all interval games with player set $N$, respectively.

For any $(N, v) \in I G^{N}$ with $i, j \in N$, let $p=\{i, j\}, M=N \backslash\{i, j\}$ and $N^{p}=M \cup\{p\}$. A reduced game of $(N, v)$ is defined as $\left(N^{p}, v^{p}\right)$ where $v^{p}(S)=v(S)$ and $v^{p}(S \cup\{p\})=v(S \cup\{i, j\})$ for any $S \in$ $2^{M}$. Note that, $v^{p}$ is the characteristic function of an interval game with $|N|-1$ players which are obtained by merging the players $i$ and $j$ into one player termed as $p$.

For any $v_{1}, v_{2} \in I G^{N}$ and $\lambda \in \mathbb{R}$, define that

- $\quad\left(v_{1}+v_{2}\right)(S)=v_{1}(S) \oplus v_{2}(S)$ for any $S \in 2^{N}$;

- $\quad\left(v_{1}-v_{2}\right)(S)=v_{1}(S) \ominus v_{2}(S)$ for any $S \in 2^{N}$;

- $\quad\left(\lambda v_{1}\right)(S)=\lambda v_{1}(S)$ for any $S \in 2^{N}$.

The following special notions proposed in [14] will play a crucial role in analysing the interval games in the sequel. For any $v \in I G^{N}$, suppose that

- $\quad v$ is uncertainty-free if $|v(S)|=0$ for any $S \in 2^{N}$;

- $\quad v$ is $[0,0]$-indifferent if $v(S) \sim[0,0]$ for any $S \in 2^{N}$;

- $\quad v_{m}$ is the median game with respect to $v$ if $v_{m}(S)=\left[\frac{\underline{v(S)}+\overline{v(S)}}{2}, \underline{\underline{v(S)}+\overline{v(S)}}\right]$ for any $S \in 2^{N}$;

- $\quad v_{u}$ is the uncertainty-spread game with respect to $v$ if $v_{u}(S)=\left[-\frac{|v(S)|}{2}, \frac{|v(S)|}{2}\right]$ for any $S \in 2^{N}$.

Denote the set of all uncertainty-free interval games in $I G^{N}$ by $U I G^{N}$ and the set of all $[0,0]$-indifferent interval games by $Z I G^{N}$. It can be easily verified that for any $v \in I G^{N}, v=v_{m}+v_{u}$ where $v_{m} \in U I G^{N}$ and $v_{u} \in Z I G^{N}$.

Let $v \in I G^{N}$ and $T \in 2^{N} \backslash\{\varnothing\}$. Define

- $\quad\left(N, u_{T}^{f}\right)$ based on $T$ as

$$
u_{T}^{f}(S)= \begin{cases}{[1,1],} & \text { if } T \subseteq S \\ {[0,0],} & \text { otherwise }\end{cases}
$$

- $\quad\left(N, z_{T}^{v_{u}(T)}\right)$ based on $T$ as

$$
z_{T}^{v_{u}(T)}(S)=\left\{\begin{array}{cc}
v_{u}(T), & \text { if } T=S \\
{[0,0],} & \text { otherwise }
\end{array}\right.
$$

Clearly, $u_{T}^{f} \in U I G^{N}$ and $z_{T}^{v_{u}(T)} \in Z I G^{N}$ for any $T \in 2^{N} \backslash\{\varnothing\}$.

Lemma 1. For any $v \in I G^{N}$, there is a sequence of real numbers $\left\{\beta_{T}\right\}_{T \in 2^{N} \backslash\{\varnothing\}}$ such that $v=$ $\sum_{T \in 2^{N} \backslash\{\varnothing\}}\left\{\beta_{T} u_{T}^{f}+z_{T}^{v_{u}(T)}\right\}$.

Proof. For any $v \in I G^{N}$, we get $v=v_{m}+v_{u}$. Since $v_{m} \in U I G^{N}$ is equivalent to a classical cooperative game, we have that $\left\{u_{T}^{f}\right\}_{T \in 2^{N} \backslash\{\varnothing\}}$ is a basis for $U I G^{N}$. Accordingly, there is a sequence of real numbers $\left\{\beta_{T}\right\}_{T \in 2^{N} \backslash\{\varnothing\}}$ such that $v_{m}=\sum_{T \in 2^{N} \backslash\{\varnothing\}} \beta_{T} u_{T}^{f}$. Moreover, $v_{u}=\sum_{T \in 2^{N} \backslash\{\varnothing\}} z_{T}^{v_{u}(T)}$ for any $v_{u} \in Z I G^{N}$. Thus, we have $v=\sum_{T \in 2^{N} \backslash\{\varnothing\}}\left\{\beta_{T} u_{T}^{f}+z_{T}^{v_{u}(T)}\right\}$. 
Let $I(\mathbb{R})^{N}$ be the set of $n$-dimensional vectors with each component in $I(\mathbb{R})$ where $n$ is the cardinality of $N$. An interval value on $I G^{N}$ is a function $\Psi: I G^{N} \rightarrow I(\mathbb{R})^{N}$ where $n=|N|$ and the $i$-th coordinate of $\Psi(v)$ represents the payoff for player $i$ in $N$.

Two interval values $\Psi$ and $\Psi^{*}$ are said to be median-indifferent to each other, denoted by $\Psi \sim \Psi^{*}$, if $\Psi_{i}(v) \sim \Psi_{i}^{*}(v)$ for any $v \in I G^{N}$ and any $i \in N$.

Lastly, we present a number of properties (or axioms) that an interval value may satisfy. Generally, an interval value may satisfy none of the following properties and it could also satisfy several properties simultaneously. An interval value $\Psi: I G^{N} \rightarrow I(\mathbb{R})^{N}$ is said to satisfy:

- Indifference efficiency (IEF): $\sum_{i \in N} \Psi_{i}(v) \sim v(N)$ for any $v \in I G^{N}$ (In this text, the summation operator for closed intervals is according to the addition operator $\oplus$.

- $\quad$ 2-players merging equivalence (2ME): $\Psi_{i}(v) \oplus \Psi_{j}(v)=\Psi_{p}\left(N^{p}, v^{p}\right)$ for any $v \in I G^{N}$ and $i, j \in N$ with $p=\{i, j\}$.

- Indifference 2-players merging equivalence $(I 2 M E): \Psi_{i}(v) \oplus \Psi_{j}(v) \sim \Psi_{p}\left(N^{p}, v^{p}\right)$ for any $v \in I G^{N}$ and $i, j \in N$ with $p=\{i, j\}$.

- Indifference 2-players Super Additivity $(I 2 S A): \Psi_{i}(v) \oplus \Psi_{j}(v) \precsim \Psi_{p}\left(N^{p}, v^{p}\right)$ for any $v \in I G^{N}$ and $i, j \in N$ with $p=\{i, j\}$.

- Indifference Marginal Contributions (IMC): Let $v, w \in I G^{N}$ and $i \in N$. If for any $S \subseteq N \backslash\{i\}$, $v(S \cup\{i\}) \ominus v(S) \sim w(S \cup\{i\}) \ominus w(S)$, then $\Psi_{i}(v) \sim \Psi_{i}(w)$.

- Indifference Dummy Property (IDP): $\Psi_{i}(v) \sim v(\{i\})$ for any $v \in I G^{N}$ and any I-dummy player $i$ in $v$ where $i$ is called a I-dummy player in $v$, if $v(S \cup\{i\}) \sim v(S) \oplus v(\{i\})$ for any $S \subseteq N \backslash\{i\}$.

- $\quad$ Symmetry $(S Y M): \Psi_{i}(v)=\Psi_{j}(v)$ for any $v \in I G^{N}$ and any symmetric players $i$ and $j$ in $v$ where $i$ and $j$ are called symmetric players in $v$ if $v(S \cup\{i\})=v(S \cup\{j\})$ for any $S \subseteq N \backslash\{i, j\}$.

- Additivity (ADD): $\Psi\left(v_{1}+v_{2}\right)=\Psi\left(v_{1}\right) \oplus \Psi\left(v_{2}\right)$ for any $v_{1}, v_{2} \in I G^{N}$.

- Uncertainty-removing Property (URP): $\left|\Psi_{i}(v)\right|=0$ for any $v \in I G^{N}$ and any uncertainty-removing player $i$ in $v$ where $i$ is called a uncertainty-removing player in $v$, if $|v(S \cup\{i\})|=0$ for any $S \subseteq N$.

- $\quad$ Equal Degree of Uncertainty $(E D U):\left|\Psi_{i}(v)\right|=\left|\Psi_{j}(v)\right|$ for any $v \in I G^{N}$ and any $i, j \in N$.

IEF says that the sum of the players' payoffs should be median-indifferent to the worth for grand coalition. This property is an extension of the well-known efficiency proposed by [15]. 2ME expresses that if we merge two players $i$ and $j$ into one player $p$, then the payoff for $p$ should agree with the sum of their respective payoffs, whereas I2ME requires that if we merge two players $i$ and $j$ into one player $p$, then the payoff for $p$ should be median-indifferent to the sum of their respective payoffs. Both of them are extensions of the 2-efficiency proposed in [16] for the values of classical cooperative games. I2SA claims that if we merge two players $i$ and $j$ into one player $p$, the payoff for $p$ should be weakly superior to the sum of their payoffs. This property is an extension of the 2-players super additivity in [16].

IMC requires that if player $i$ 's marginal contributions to all coalitions in two distinct games are median-indifferent to each other, then the payoffs for $i$ received in these two games should be median-indifferent to each other. IDP expresses that if a player $i$ 's marginal contribution to any coalition is median-indifferent to the worth from $i$ 's individual action, then the payoff distributed for $i$ should also be median-indifferent to the worth of $i$ 's individual action. These two properties are generalizations of the marginal contributions and of the dummy property in [17], separately. SYM and ADD are straightforward extensions of the symmetry and of the additivity in [16], respectively.

As far as we know, none property concerning the division of uncertainty degree among the players has been proposed within the framework of interval cooperative games. In view of this, we formulate the properties of EDU and of URP. EDU expresses that the uncertainty degree of each player's payoff is identical. URP says that if there exists an uncertainty-removing player who has more information or the ability to forecast payoffs of any involved coalitions accurately, then the payoff distributed for this player should be free from uncertainty. 


\section{Comparison between the Interval Banzhaf Value and the Interval Banzhaf-like Value}

In order to approach the cooperative interval games, the notion of interval Banzhaf value was proposed in [13].

The interval Banzhaf value for $v \in I G^{N}$ is given as $\mathcal{B}(v)=\left(\mathcal{B}_{1}(v), \mathcal{B}_{2}(v), \ldots, \mathcal{B}_{n}(v)\right)$ where $\mathcal{B}_{i}(v)=$ $\frac{1}{2^{|N|-1}} \sum_{S \subseteq N \backslash\{i\}}\{v(S \cup\{i\})-v(S)\}$ for any $i \in N$. As the interval subtraction operator employed in this notion is only well-defined for an ordered interval pair $(I, J)$ with $|I| \geq|J|, \mathcal{B}$ is undefinable for interval games which are not size monotonic, where a game $v \in I G^{N}$ is called a size monotonic game if $|v(S)| \leq|v(T)|$ for any $S, T \in 2^{N}$ with $S \subseteq T$. Denote $S M I G^{N}$ the class of size monotonic interval games in $I G^{N}$. To clarify this point, see the following example.

Example 1. Consider $(N, v)$ in Table 1. Due to $|v(\{1,2,3\})|<|v(\{2,3\})|$ and $|v(\{1,3\})|<|v(\{1\})|$, $v$ is not a size monotonic interval game. As neither $v(\{1,2,3\})-v(\{2,3\})$ nor $v(\{1,3\})-v(\{1\})$ is definable, $\mathcal{B}(v)$ is undefinable.

Table 1. $(\mathrm{N}, \mathrm{v})$.

\begin{tabular}{cccccccc}
\hline$S$ & $\{1\}$ & $\{2\}$ & $\{3\}$ & $\{1,2\}$ & $\{1,3\}$ & $\{2,3\}$ & $\{1,2,3\}$ \\
\hline$v(S)$ & {$[2,4]$} & {$[3,4]$} & {$[3,4]$} & {$[8,9]$} & {$[7,8]$} & {$[7,9]$} & {$[12,13]$} \\
\hline
\end{tabular}

To overcome this mentioned shortcoming of the interval Banzhaf value, we propose an alternative Banzhaf value for cooperative interval games. We term this proposal as the interval Banzhaf-like value to distinguish it from the interval Banzhaf value introduced in [13].

Definition 1. The interval Banzhaf-like value for $v \in I G^{N}$ is defined as $\mathcal{B}^{*}(v)=\left(\mathcal{B}_{1}^{*}(v), \mathcal{B}_{2}^{*}(v), \ldots, \mathcal{B}_{n}^{*}(v)\right)$ where $\mathcal{B}_{i}^{*}(v)=\frac{1}{2^{|N|-1}} \sum_{S \subseteq N \backslash\{i\}}\{v(S \cup\{i\}) \ominus v(S)\}$ for any $i \in N$.

As the interval subtraction operator used in this definition is well-defined for any interval pair $(I, J)$, the interval Banzhaf-like value is definable for every possible interval game in $I G^{N}$. Reconsider the interval game $v$ in Table 1 . We have that $\mathcal{B}^{*}(v)=\left(\left[3, \frac{21}{4}\right],\left[\frac{14}{4}, \frac{23}{4}\right],\left[3, \frac{21}{4}\right]\right)$, but $\mathcal{B}(v)$ does not exist. It is important to point out that given $v \in I G^{N}, \mathcal{B}^{*}(v)$ is not necessarily the same as $\mathcal{B}(v)$ even through both $\mathcal{B}(v)$ and $\mathcal{B}^{*}(v)$ are definable. To show this statement, we represent the example in [13] as follows.

Example 2. Consider $(N, w)$ in Table 2 which is size monotonic. $\mathcal{B}(w)=\left(\left[\frac{5}{2}, \frac{7}{2}\right],\left[\frac{9}{2}, \frac{11}{2}\right],[5,6]\right)$ whereas $\mathcal{B}^{*}(w)=\left(\left[\frac{3}{2}, \frac{9}{2}\right],\left[\frac{7}{2}, \frac{13}{2}\right],[4,7]\right)$.

Table 2. $(\mathrm{N}, \mathrm{w})$.

\begin{tabular}{cccccccc}
\hline$S$ & $\{1\}$ & $\{2\}$ & $\{3\}$ & $\{1,2\}$ & $\{1,3\}$ & $\{2,3\}$ & $\{1,2,3\}$ \\
\hline$w(S)$ & {$[2,3]$} & {$[3,4]$} & {$[4,5]$} & {$[6,8]$} & {$[6,8]$} & {$[9,11]$} & {$[12,15]$} \\
\hline
\end{tabular}

The next theorem reveals the relation between the interval Banzhaf-like value and the interval Banzhaf value.

Theorem 1. $\mathcal{B}^{*}(v) \sim \mathcal{B}(v)$ for any $v \in S M I G^{N}$.

Proof. For any $v \in S M I G^{N}, i \in N$ and $S \subseteq N \backslash\{i\}$, we have 


$$
\begin{aligned}
v(S \cup\{i\}) \ominus v(S) & =[v \underline{v(S \cup\{i\})}-\overline{v(S)}, \overline{v(S \cup\{i\})}-\underline{v(S)}] \\
& \sim\left[\frac{\underline{v(S \cup\{i\})+\overline{v(S \cup\{i\})}}}{2}-\frac{v(S)+\overline{v(S)}}{2}, \frac{v(S \cup\{i\})+\overline{v(S \cup\{i\})}}{2}-\frac{v(S)+\overline{v(S)}}{2}\right] \\
& \sim[\underline{v(S \cup\{i\})}-\underline{v(S)}, \overline{v(S \cup\{i\})}-\overline{v(S)}]=v(S \cup\{i\})-v(S) .
\end{aligned}
$$

Moreover, for any $i \in N$, we get that $\sum_{S \subseteq N \backslash\{i\}}\{v(S \cup\{i\}) \ominus v(S)\} \sim \sum_{S \subseteq N \backslash\{i\}}\{v(S \cup\{i\})-v(S)\}$ and $\frac{1}{2^{|N|-1}} \sum_{S \subseteq N \backslash\{i\}}\{v(S \cup\{i\}) \ominus v(S)\} \sim \frac{1}{2^{|N|-1}} \sum_{S \subseteq N \backslash\{i\}}\{v(S \cup\{i\})-v(S)\}$. Thus, $\mathcal{B}^{*}(v) \sim \mathcal{B}(v)$.

Note that, both the interval Banzhaf-like value and the interval Banzhaf value coincide with the Banzhaf value in [16] whenever a cooperative interval game degenerates into a classical cooperative game.

\section{The Properties for the Interval Banzhaf-like Value and its Median-Indifference Values}

The next proposition shows the properties that the interval Banzhaf-like value is provided with.

Proposition 1. The interval Banzhaf-like value $\mathcal{B}^{*}$ satisfies I2ME, IDP, SYM, IMC, ADD and EDU.

Proof. It is easy to prove that $\mathcal{B}^{*}$ satisfies SYM and ADD.

Take any $v \in I G^{N}$ and any $i, j \in N$ with $p=\{i, j\}$. Then

$$
\begin{aligned}
& \mathcal{B}_{p}^{*}\left(v^{p}\right)=\frac{1}{2^{|N|-2}} \sum_{S \subseteq N \backslash\{p\}}\left\{v^{p}(S \cup\{p\}) \ominus v^{p}(S)\right\}=\frac{1}{2^{|N|-2}} \sum_{S \subseteq N \backslash\{i, j\}}\{v(S \cup\{i, j\}) \ominus v(S)\} . \\
& \mathcal{B}_{i}^{*}(v)=\frac{1}{2^{|N|-1}} \sum_{S \subseteq N \backslash\{i\}}\{v(S \cup\{i\}) \ominus v(S)\} \\
&=\frac{1}{2^{|N|-1}} \sum_{S \subseteq N \backslash\{i, j\}}\{v(S \cup\{i, j\}) \ominus v(S \cup\{j\}) \oplus v(S \cup\{i\}) \ominus v(S)\} . \\
& \mathcal{B}_{j}^{*}(v)=\frac{1}{2^{|N|-1}} \sum_{S \subseteq N \backslash\{j\}}\{v(S \cup\{j\}) \ominus v(S)\} \\
&=\frac{1}{2^{|N|-1}} \sum_{S \subseteq N \backslash\{i, j\}}\{v(S \cup\{i, j\}) \ominus v(S \cup\{i\}) \oplus v(S \cup\{j\}) \ominus v(S)\} . \\
& \mathcal{B}_{i}^{*}(v) \oplus \mathcal{B}_{j}^{*}(v)=\frac{1}{2^{|N|-1}} \sum_{S \subseteq N \backslash\{i, j\}} 2\{v(S \cup\{i, j\}) \ominus v(S)\} \\
& \oplus \frac{1}{2^{|N|-1}} \sum_{S \subseteq N \backslash\{i, j\}}\{v(S \cup\{i\}) \ominus v(S \cup\{i\})\} \\
& \oplus \frac{1}{2^{|N|-1}} \sum_{S \subseteq N \backslash\{i, j\}}\{v(S \cup\{j\}) \ominus v(S \cup\{j\})\} \\
& \sim \frac{1}{2^{|N|-1}} \sum_{S \subseteq N \backslash\{i, j\}} 2\{v(S \cup\{i, j\}) \ominus v(S)\} \\
&=\frac{1}{2^{|N|-2}} \sum_{S \subseteq N \backslash\{i, j\}}\{v(S \cup\{i, j\}) \ominus v(S)\} \\
&=\frac{1}{2^{|N|-2}} \sum_{S \subseteq N^{p} \backslash\{p\}}\left\{v p(S \cup\{p\}) \ominus v^{p}(S)\right\}=\mathcal{B}_{p}^{*}\left(v^{p}\right) .
\end{aligned}
$$

Thus, $\mathcal{B}^{*}$ satisfies I2ME.

Let $v \in I G^{N}$ and $i$ be an I-dummy player in $v$. For any $S \subseteq N \backslash\{i\}$, we get $\frac{1}{2}\{\overline{v(S \cup\{i\})}+\underline{v(S \cup\{i\})}\}=\frac{1}{2}\{\overline{v(S)}+\underline{v(S)}+\overline{v(\{i\})}+\underline{v(\{i\})}\}$ and $v(S \cup\{i\}) \ominus \bar{v}(S)=[\underline{v(S \cup\{i\})}-$ $\overline{v(S)}, \overline{v(S \cup\{i\})}-\underline{v(S)]} \sim[\underline{v(\{i\})}, \overline{v(\{i\})}]$. Then, $\mathcal{B}_{i}^{*} \overline{(v)}=\frac{1}{2^{|N|-1}} \sum_{S \subseteq N \backslash\{i\}}\{v(S \cup\{i\}) \overline{\ominus v(S)\}} \sim$ $[v(\{i\}), \overline{v(\{i\})}]$. Thus, $\mathcal{B}^{*}$ satisfies IDP.

Let $v, w \in I G^{N}$ and $i \in N$. If $\{v(S \cup\{i\}) \ominus v(S)\} \sim\{w(S \cup\{i\}) \ominus w(S)\}$ for any $S \subseteq N \backslash\{i\}$, then we get $\mathcal{B}^{*}{ }_{i}(v)=\frac{1}{2^{|N|-1}} \sum_{S \subseteq N \backslash\{i\}}(v(S \cup\{i\}) \ominus v(S)) \sim \frac{1}{2^{|N|-1}} \sum_{S \subseteq N \backslash\{i\}}(w(S \cup\{i\}) \ominus w(S))=\mathcal{B}^{*}{ }_{i}(w)$. Thus, $\mathcal{B}^{*}$ satisfies IMC. 
Lastly, we prove that $\mathcal{B}^{*}$ satisfies EDU. For any $v \in I G^{N}$ and $i \in N$, we have

$\left|\mathcal{B}_{i}^{*}(v)\right|=\frac{1}{2^{|N|-1}} \sum_{S \subseteq N \backslash\{i\}}|v(S \cup\{i\}) \ominus v(S)|=\frac{1}{2^{|N|-1}} \sum_{S \subseteq N \backslash\{i\}}|v(S \cup\{i\})|+|v(S)|=\frac{1}{2^{|N|-1}} \sum_{S \subseteq N}|v(S)|$.

Thus, $\left|\mathcal{B}_{i}^{*}(v)\right|=\left|\mathcal{B}_{j}^{*}(v)\right|$ for any $i, j \in N$.

Remark 1. $\mathcal{B}^{*}$ satisfies I2SA, but violates IEF, $2 M E$ and URP.

As I2ME is a stronger property than I2SA, $\mathcal{B}^{*}$ satisfies I2SA. To verify the second point in Remark 1 , consider the following example.

Example 3. Reconsider $(N, v)$ in Example 1. We have that $\sum_{i \in N} \mathcal{B}_{i}^{*}(v)=\left[\frac{19}{2}, \frac{65}{4}\right]$, whereas $v(N)=[12,13]$. Clearly, $\sum_{i \in N} \mathcal{B}_{i}^{*}(v) \sim v(N)$ does not hold. Amalgamating the players 1 and 2 into one player $p$, a reduced interval game can be obtained as described in Table 3. We have that $\mathcal{B}_{p}^{*}(w)=[8,9.5]$, whereas $\mathcal{B}_{1}^{*}(v) \oplus \mathcal{B}_{2}^{*}(v)=$ $\left[\frac{13}{2}, 11\right]$.

Consider $(N, v)$ in Table 4. We get $\mathcal{B}^{*}(v)=\left(\left[\frac{7}{2}, 5\right],\left[\frac{9}{2}, 6\right],\left[\frac{9}{2}, 6\right]\right)$. Player 1 is an uncertainty-removing player in v. However, $\left|\mathcal{B}_{1}^{*}(v)\right| \neq 0$.

Table 3. $\left(N^{p}, w\right)$.

\begin{tabular}{cccc}
\hline$S$ & $\{\mathrm{p}\}$ & $\{3\}$ & $\{\mathrm{p}, 3\}$ \\
\hline $\boldsymbol{w}(S)$ & {$[8,9]$} & {$[3,4]$} & {$[12,13]$} \\
\hline
\end{tabular}

Table 4. $(\mathrm{N}, \mathrm{v})$.

\begin{tabular}{cccccccc}
\hline$S$ & $\{1\}$ & $\{2\}$ & $\{3\}$ & $\{1,2\}$ & $\{1,3\}$ & $\{2,3\}$ & $\{1,2,3\}$ \\
\hline$v(S)$ & {$[3,3]$} & {$[2,4]$} & {$[2,4]$} & {$[7,7]$} & {$[7,7]$} & {$[8,10]$} & {$[15,15]$} \\
\hline
\end{tabular}

In contrast, it can be verified that the interval Banzhaf value satisfies 2ME, IDP, SYM, IMC and ADD, but violates both IEF and EDU over the class of size monotonic interval games.

Generally, for a given interval game, there exist more than one interval values that are median-indifferent to the Banzhaf-like value. Thus, it is meaningful to find out the conditions for the determination of these values.

Theorem 2. If an interval value $\Psi: I G^{N} \rightarrow I(\mathbb{R})^{N}$ satisfies I2ME, IDP, SYM and IMC, then $\Psi(v) \sim \mathcal{B}^{*}(v)$ for any $v \in I G^{N}$.

Proof. As $v \sim v_{m}$ for any $v \in I G^{N}$, and both $\Psi$ and $\mathcal{B}^{*}$ satisfy IMC, we get $\Psi(v) \sim \Psi\left(v_{m}\right)$ and $\mathcal{B}^{*}(v) \sim \mathcal{B}^{*}\left(v_{m}\right)$. Thus, in order to prove $\Psi(v) \sim \mathcal{B}^{*}(v)$, it is sufficient to prove $\Psi\left(v_{m}\right) \sim \mathcal{B}^{*}\left(v_{m}\right)$.

Step 1: Let $|T|=t$. If $t \leq 2$, then by I2ME, SYM and IDP, we have that $\Psi_{i}\left(u_{T}^{f}\right) \sim\left[\frac{1}{2^{|T|-1}}, \frac{1}{2^{|T|-1}}\right]$ if $i \in$ $T$, and that $\Psi_{i}\left(u_{T}^{f}\right) \sim[0,0]$ if $i \notin T$. Suppose that the above statement holds for $|T|=t \leq m$ (where $m$ is an integer and $m>2$ ). Consider the case with $|T|=t=m+1$. For any $i, j \in T$, let $p=\{i, j\}$. Take the reduced game $\left(N^{p}, v^{p}\right)$ where $v=u_{T}^{f}$. By the above induction hypothesis, we get $\Psi_{p}\left(v^{p}\right) \sim\left[\frac{1}{2^{t-1}}, \frac{1}{2^{t-1}}\right]$. Since $\Psi$ satisfies I2ME and SYM, we have that $\Psi_{i}(v)=\Psi_{j}(v) \sim\left[\frac{1}{2^{t}}, \frac{1}{2^{t}}\right]=$ $\mathcal{B}_{i}^{*}\left(u_{T}^{f}\right)=\mathcal{B}_{j}^{*}\left(u_{T}^{f}\right)$. As $\Psi$ satisfies IDP and any $i \in N \backslash T$ is an I-dummy player in $u_{T}^{f}$, we have that $\Psi_{i}(v) \sim[0,0]=\mathcal{B}_{i}^{*}\left(u_{T}^{f}\right)$. Therefore, $\Psi\left(u_{T}^{f}\right) \sim \mathcal{B}^{*}\left(u_{T}^{f}\right)$ for any $T \in 2^{N} \backslash\{\varnothing\}$. Similarly, we may obtain that $\Psi\left(\beta u_{T}^{f}\right) \sim \mathcal{B}^{*}\left(\beta u_{T}^{f}\right)$ for any $\beta \in \mathbb{R}$ and $T \in 2^{N} \backslash\{\varnothing\}$.

Step 2: Let $v \in I G^{N}$. By Lemma 1, there exists a unique sequence of $\left\{\beta_{T}^{f}\right\}_{T \in 2^{N} \backslash\{\varnothing\}}$ (where $\beta_{T}^{f} \in \mathbb{R}$ ) such that $v_{m}=\sum_{T \in 2^{N} \backslash\{\varnothing\}} \beta_{T}^{f} u_{T}^{f}$. Then, take $Z_{c}\left(v_{m}\right)=\left\{T \in 2^{N} \backslash\{\varnothing\} \mid v_{m}=\sum_{T \in 2^{N} \backslash\{\varnothing\}} \beta_{T}^{f} u_{T}^{f}\right.$ and $\beta_{T}^{f} \neq$ 
$0\}$. For any $v \in I G^{N}$ with $\left|Z_{c}\left(v_{m}\right)\right|=1$, there exists a unique $\beta \in \mathbb{R}$ such that $v_{m}=\beta u_{T}^{f}$. Based on the conclusion in Step 1, we have $\Psi\left(v_{m}\right) \sim \mathcal{B}^{*}\left(v_{m}\right)$.

Suppose that $\Psi\left(v_{m}\right) \sim \mathcal{B}^{*}\left(v_{m}\right)$ for any $(N, v) \in I G^{N}$ with $|N| \leq n$, and that $\Psi\left(v_{m}\right) \sim \mathcal{B}^{*}\left(v_{m}\right)$ for $(N, v) \in I G^{N}$ with $|N|=n+1$ if $\left|Z_{c}\left(v_{m}\right)\right| \leq k$ (where $k \geq 1$ and $k$ is a positive integer). Consider any $v \in I G^{N}$ with $|N|=n+1$ and $\left|Z_{\mathcal{c}}\left(v_{m}\right)\right|=k+1$. Then, there exists a sequence of coalitions $T_{1}, \ldots, T_{k+1}$ such that $v_{m}=\sum_{r=1}^{k+1} \beta_{T_{r}}^{f} u_{T_{r}}^{f}$. Let $T=T_{1} \cap \ldots \cap T_{k+1}$. Due to $k+1 \geq 2$, we get $N \backslash T \neq \varnothing$.

(i) Take $i \in N \backslash T$ and $\left(N, v_{m}^{\prime}\right)$ where $v_{m}^{\prime}=\sum_{r: i \in T_{r}, 1 \leq r \leq k+1} \beta_{T_{r}}^{f} u_{T_{r}}^{f}$. Due to $\left|Z_{c}\left(v_{m}^{\prime}\right)\right| \leq k$, we get $\Psi\left(v_{m}^{\prime}\right) \sim \mathcal{B}^{*}\left(v_{m}^{\prime}\right)$. Moreover, it can be verified that $v_{m}(S \cup\{i\}) \ominus v_{m}(S)=v_{m}^{\prime}(S \cup\{i\}) \ominus v_{m}^{\prime}(S)$ for any $S \subseteq N \backslash\{i\}$. As both $\Psi$ and $\mathcal{B}$ satisfy IMC, we get $\Psi_{i}\left(v_{m}\right) \sim \Psi_{i}\left(v_{m}^{\prime}\right)$ and $\mathcal{B}_{i}^{*}\left(v_{m}\right) \sim \mathcal{B}_{i}^{*}\left(v_{m}^{\prime}\right)$. Because of $\Psi\left(v_{m}^{\prime}\right) \sim \mathcal{B}^{*}\left(v_{m}^{\prime}\right)$ implying $\Psi_{i}\left(v_{m}^{\prime}\right) \sim \mathcal{B}_{i}^{*}\left(v_{m}^{\prime}\right)$, we have that $\Psi_{i}\left(v_{m}\right) \sim \mathcal{B}_{i}^{*}\left(v_{m}\right)$ for each $i \in N \backslash T$. (ii) Take $j \in T$ and amalgamate $j$ with the given $i \in N \backslash T$. Put $p=\{i, j\}$ and consider $v_{m}^{p}$ which is the median game of $v^{p}$. According to the former induction hypothesis, we have $\Psi_{p}\left(v_{m}^{p}\right) \sim \mathcal{B}_{p}^{*}\left(v_{m}^{p}\right)$. As both $\Psi$ and $\mathcal{B}^{*}$ satisfy I2ME, we may conclude that $\Psi_{p}\left(v_{m}^{p}\right) \sim\left(\Psi_{i}\left(v_{m}\right) \oplus \Psi_{j}\left(v_{m}\right)\right)$ and $\mathcal{B}_{p}^{*}\left(v_{m}^{p}\right) \sim$ $\left(\mathcal{B}_{i}^{*}\left(v_{m}\right) \oplus \mathcal{B}_{j}^{*}\left(v_{m}\right)\right)$, which implies $\Psi_{j}\left(v_{m}\right) \sim \mathcal{B}_{j}^{*}\left(v_{m}\right)$. Therefore, $\Psi\left(v_{m}\right) \sim \mathcal{B}^{*}\left(v_{m}\right)$ for any $v \in I G^{N}$ with $|N|=n+1$ and $\left|Z_{c}\left(v_{m}\right)\right|=k+1$.

To sum up, we may conclude that $\Psi(v) \sim \mathcal{B}^{*}(v)$ for any $v \in I G^{N}$.

Remark 2. Theorem 2 does not hold anymore if I2ME is replaced by I2SA without changing other properties.

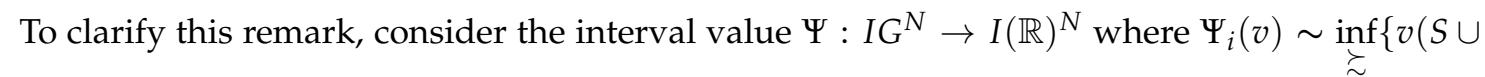
$\{i\}) \ominus v(S)\}$ for any $i \in N$ with $S \subseteq N \backslash\{i\}$. It can be proved that $\Psi$ satisfies I2SA, IDP, SYM, IMC. However, $\Psi \sim \mathcal{B}^{*}$ does not hold.

Since the interval value that satisfies the mentioned properties is not unique, it is interesting to further narrow down the category of these interval values. The next theorem shows that a more specific kind of interval values can be identified by replacing I2ME and IMC in Theorem 2 with I2SA, ADD and EDU.

Theorem 3. If an interval value $\Psi: I G^{N} \rightarrow I(\mathbb{R})^{N}$ satisfies I2SA, IDP, SYM, ADD and EUC, then $\Psi(v) \sim \mathcal{B}^{*}(v)$ for any $v \in I G^{N}$. Moreover, given a $v \in I G^{N}$, there exists a real number $\alpha \in \mathbb{R}$ such that $\left|\Psi_{i}(v)\right|=\alpha\left|\mathcal{B}_{i}^{*}(v)\right|$ for any $i \in N$.

Proof. Take any $v \in I G^{N}$. Since $v=v_{m} \oplus v_{u}$, and by the fact that $\Psi$ and $\mathcal{B}^{*}$ satisfy ADD, we have that $\Psi(v)=\Psi\left(v_{m}\right) \oplus \Psi\left(v_{u}\right)$ and $\mathcal{B}^{*}(v)=\mathcal{B}^{*}\left(v_{m}\right) \oplus \mathcal{B}^{*}\left(v_{u}\right)$. In order to prove that $\Psi(v) \sim \mathcal{B}^{*}(v)$, it is sufficient to prove that $\Psi\left(v_{m}\right) \sim \mathcal{B}^{*}\left(v_{m}\right)$ and $\Psi\left(v_{u}\right) \sim \mathcal{B}^{*}\left(v_{u}\right)$ both hold.

As $v_{u}(S) \sim[0,0]$ for any $S \in 2^{N} \backslash\{\varnothing\}$, we may conclude that any $i \in N$ is an I-dummy player in $v_{u}$. Since both $\Psi$ and $\mathcal{B}^{*}$ satisfy IDP, we get $\Psi\left(v_{u}\right) \sim \mathcal{B}^{*}\left(v_{u}\right)$. The next step is to prove that $\Psi\left(v_{m}\right) \sim \mathcal{B}^{*}\left(v_{m}\right)$. Let $S, T \in 2^{N} \backslash\{\varnothing\}$ and $k$ be a nonnegative integer. Define $g_{T}^{\{k ; n\}} \in I G^{N}$ with $n$ is the cardinality of $N$ and

$$
g_{T}^{\{k ; n\}}(S)=\left\{\begin{array}{lc}
{[1,1]} & \text { if } k \leq|T \cap S| \\
{[0,0]} & \text { otherwise. }
\end{array}\right.
$$

Suppose $\Psi$ is an interval value on $I G^{N}$ satisfying I2SA, IDP, SYM, ADD and EDU. Then, for any $u_{T}^{f} \in U I G^{N}$, we have

$$
\Psi\left((|T|-1) u_{T}^{f}\right) \oplus \Psi\left(g_{T}^{\{|T|-1 ; n\}}\right)=\sum_{T^{\prime}: T^{\prime} \subset T,\left|T^{\prime}\right|=|T|-1} \Psi\left(u_{T^{\prime}}^{f}\right) .
$$

As both $\Psi$ and $\mathcal{B}^{*}$ satisfy IDP, it can be verified that $\Psi\left(v_{m}\right) \sim \mathcal{B}^{*}\left(v_{m}\right)$ for any $v \in I G^{N}$ with $|N|=$ 1 ; and that $\Psi\left(u_{T}^{f}\right) \sim \mathcal{B}^{*}\left(u_{T}^{f}\right)$ for any $u_{T}^{f} \in U I G^{N}$ with $|T|=1$. 
Step 1: Suppose that $\Psi\left(u_{T}^{f}\right) \sim \mathcal{B}^{*}\left(u_{T}^{f}\right)$ for any $u_{T}^{f} \in U I G^{N}$ with $|N| \leq n$, and that $\Psi\left(u_{T}^{f}\right) \sim \mathcal{B}^{*}\left(u_{T}^{f}\right)$ for any $u_{T}^{f} \in U I G^{N}$ with $|T| \leq k$ (where $k \geq 1$ ) and $|N|=n+1$. Then we get

$$
\left\{\begin{array}{cc}
\Psi_{i}\left(u_{T}^{f}\right) \sim\left[\frac{1}{2^{|T|-1}}, \frac{1}{2^{|T|-1}}\right], & \text { if } i \in T \\
\Psi_{i}\left(u_{T}^{f}\right) \sim[0,0], & \text { otherwise. }
\end{array}\right.
$$

As $\Psi$ satisfies ADD, by Lemma 1, we get that $\Psi\left(v_{m}\right) \sim \mathcal{B}^{*}\left(v_{m}\right)$ for any $v \in I G^{N}$ with $|N| \leq n$.

Step 2: Let $u_{T}^{f} \in U I G^{N}$ with $|N|=n+1$ and $|T|=k+1$. As $\Psi$ satisfies IDP and SYM, there exist two intervals $a=[\underline{a}, \bar{a}]$ and $b=[\underline{b}, \bar{b}]$ satisfying the following conclusions:

$$
\begin{gathered}
\left\{\begin{array}{c}
\Psi_{i}\left(u_{T}^{f}\right) \sim[\underline{a}, \bar{a}] \text { if } i \in T ; \\
\Psi_{i}\left(u_{T}^{f}\right) \sim[0,0] \text { if } i \notin T .
\end{array}\right. \\
\left\{\begin{array}{l}
\Psi_{i}\left(g_{T}^{\{k ; n+1\}}\right) \sim[\underline{b}, \bar{b}] \text { if } i \in T ; \\
\Psi_{i}\left(g_{T}^{\{k ; n+1\}}\right) \sim[0,0] \text { if } i \notin T .
\end{array}\right.
\end{gathered}
$$

By combining the (1), (2), (3) and the induction hypothesis, we get that

$$
k[\underline{a}, \bar{a}]+[\underline{b}, \bar{b}]=[k \underline{a}+\underline{b}, k \bar{a}+\bar{b}] \sim\left[\frac{|T|-1}{2^{k-1}}, \frac{|T|-1}{2^{k-1}}\right]=\left[\frac{k}{2^{k-1}}, \frac{k}{2^{k-1}}\right] .
$$

(i) Let $i, j \in T$ and $p=\{i, j\}$. Amalgamating $i$ to $j$ in $u_{T^{\prime}}^{f}$, we derive $u_{T^{\prime}}^{f, n}$ (as above $T^{\prime}=T \backslash\{i\}$ ). By I2SA, we have

$$
[2 \underline{a}, 2 \bar{a}] \precsim\left[\frac{1}{2^{k-1}}, \frac{1}{2^{k-1}}\right] .
$$

(ii) Let $i, j \in T$ and $p=\{i, j\}$. By amalgamating $i$ to $j$ in $g_{T}^{\{k ; n+1\}}$, we get $\left(N^{\prime}, g_{T^{\prime}}^{\{k ; n\}}\right)$ in which $N^{\prime}=N \backslash\{i, j\} \cup\{p\}, T^{\prime}=T \backslash\{i, j\} \cup\{p\}$ and

$$
g_{T^{\prime}}^{\{k ; n\}}=\left\{\begin{array}{cc}
{[1,1],} & \text { if } p \in S \text { and } k-2 \leq|(S \backslash\{p\}) \cap T| \\
{[0,0],} & \text { otherwise. }
\end{array}\right.
$$

Due to $\left|N^{\prime}\right|=n$ and $\left|T^{\prime}\right|=k$, by the induction hypothesis and (3), we have

$$
\Psi_{p}\left(g_{T^{\prime}}^{\{k n\}}\right)=\left[\frac{k}{2^{k-1}}, \frac{k}{2^{k-1}}\right] \text { and }[2 \underline{b}, 2 \bar{b}] \precsim\left[\frac{k}{2^{k-1}}, \frac{k}{2^{k-1}}\right] .
$$

According to (5) and (6), we have

$$
[k \underline{a}+\underline{b}, k \bar{a}+\bar{b}] \precsim\left[\frac{k}{2^{k}}+\frac{k}{2^{k}}, \frac{k}{2^{k}}+\frac{k}{2^{k}}\right]=\left[\frac{k}{2^{k-1}}, \frac{k}{2^{k-1}}\right] .
$$

According to (4) and (7), we have $[\underline{a}, \bar{a}] \sim\left[\frac{1}{2^{k}}, \frac{1}{2^{k}}\right]$. By means of ADD, we get that for any $c \geq 0$, $|N|=n+1$ and $T \in 2^{N} \backslash\{\varnothing\}$,

$$
\left\{\begin{array}{c}
\Psi_{i}\left(c \cdot u_{T}^{f}\right) \sim\left[\frac{c}{2^{|T|-1}}, \frac{c}{2^{|T|-1}}\right], \text { if } i \in T \\
\Psi_{i}\left(c \cdot u_{T}^{f}\right) \sim[0,0], \text { if } i \notin T .
\end{array}\right.
$$

Thus, we have $\Psi\left(v_{m}\right) \sim \mathcal{B}^{*}\left(v_{m}\right)$ for any $v \in I G^{N}$ with $|N|=n+1$.

To sum up, we may conclude that for any $v \in I G^{N} \Psi\left(v_{m}\right) \sim \mathcal{B}^{*}\left(v_{m}\right)$ and $\Psi\left(v_{u}\right) \sim \mathcal{B}^{*}\left(v_{u}\right)$, which implies $\Psi(v) \sim \mathcal{B}^{*}(v)$. As $\Psi$ satisfies EDU, we get that $\left|\Psi_{i}(v)\right|=\left|\Psi_{j}(v)\right|$ for any $i, j \in N$. According to Proposition 1, there exists a real number $\alpha \in \mathbb{R}$ such that $\left|\Psi_{i}(v)\right|=\alpha\left|\mathcal{B}_{i}^{*}(v)\right|$. 
As showed in Proposition 1, the interval Banzhaf-like value satisfies ADD and EDU. Thus we may conclude that $\mathcal{B}_{i}^{*}=\mathcal{B}_{i}^{*}\left(v_{m}\right) \oplus \frac{1}{2^{|N|-1}} \sum_{S \subseteq N} v_{u}(S)$ for any $i \in N$. Theorem 3 expresses an important fact that the fundamental differences between $\mathcal{B}^{*}$ and the other interval values that satisfy the mentioned properties in Theorem 3 are the sizes of uncertainty distributed among players.

It is important to mention that neither Theorem 2 nor Theorem 3 holds in reverse direction. To clarify this point, we present two counterexamples as follows.

(1) Take $\Psi: I G^{N} \rightarrow I(\mathbb{R})^{N}$ where $\Psi_{i}(v)=\mathcal{B}_{i}^{*}\left(v_{m}\right) \oplus[-i, i]$ for any $v \in I G^{N}, N=\{1,2, \ldots, n\}$ and $i \in N$. It can be verified that $\Psi \sim \mathcal{B}^{*}$, but violates the property of SYM.

(2) Take $\Psi^{\prime}: I G^{N} \rightarrow I(\mathbb{R})^{N}$ where $\Psi_{i}^{\prime}(v)=\mathcal{B}_{i}^{*}\left(v_{m}\right) \oplus\left[-\inf _{j \in N}\{|v(j)|\}, \inf _{j \in N}\{|v(j)|\}\right]$ for any $v \in I G^{N}$ and $i, j \in N$. It can be verified that $\Psi^{\prime} \sim \mathcal{B}^{*}$. Moreover, there exists $\alpha \in \mathbb{R}$ such that $\left|\Psi_{i}^{\prime}(v)\right|=$ $\alpha\left|\mathcal{B}_{i}^{*}(v)\right|$ for any $i \in N$. However, $\Psi^{\prime}$ fails to satisfy the property of ADD.

In order to compare the properties in Theorem 2 and properties in Theorem 3, we propose the Remark 3.

Remark 3. If an interval value $\Psi: I G^{N} \rightarrow I(\mathbb{R})^{N}$ satisfies I2SA,IDP, SYM, ADD and EDU, then $\Psi$ satisfies I2ME, IDP, SYM, and IMC, but not vice versa.

Proof. Assume that $\Psi: I G^{N} \rightarrow I(\mathbb{R})^{N}$ satisfies I2SA, IDP, SYM and ADD.

By Theorem 3, we get that $\Psi_{i}(v) \sim \mathcal{B}_{i}^{*}(v)$ for any $v \in I G^{N}$ and $i \in N$. Let $v \in I G^{N}$ and $i, j \in N$. By amalgamating $i, j \in N$ into $p=\{i, j\}$, we obtain $\left(N^{p}, v^{p}\right)$. Then $\Psi_{p}\left(v^{p}\right) \sim \mathcal{B}_{p}^{*}\left(v^{p}\right)$. Since $\mathcal{B}^{*}$ satisfies I2ME, we have $\Psi_{p}\left(v^{p}\right) \sim \mathcal{B}_{p}^{*}\left(v^{p}\right) \sim\left(\mathcal{B}_{i}^{*}(v) \oplus \mathcal{B}_{j}^{*}(v)\right) \sim\left(\Psi_{i}(v) \oplus \Psi_{j}(v)\right)$. Thus, $\Psi$ satisfies I2ME.

Let $v, w \in I G^{N}$. Let $i, j \in N$ with that $(v(S \cup\{i\}) \ominus v(S)) \sim(w(S \cup\{i\}) \ominus w(S))$ for any $S \in 2^{N}$. As $\mathcal{B}^{*}$ satisfies IMC, we have $\mathcal{B}_{i}^{*}(v) \sim \mathcal{B}_{i}^{*}(w)$ for any $i \in N$. As $\Psi(v) \sim \mathcal{B}^{*}(v)$ for any $v \in I G^{N}$, we have $\Psi(v) \sim \Psi(w)$.

Let $v \in I G^{N}$ and $D(N, v)=\{i \in N \mid$ there exists a $j \in N \backslash$ $\{i\}$ such that $i$ and $j$ are symmetric in $v\}$. Define $\Psi: I G^{N} \rightarrow I(\mathbb{R})^{N}$ as that for any $v \in I G^{N}$, $\Psi_{i}(v)=\mathcal{B}_{i}^{*}\left(v_{m}\right) \oplus I_{i}^{\circ}$ where $I_{i}^{\circ}=[-1,1]$ if $i \in D(N, v)$ and $I_{i}^{\circ}=[0,0]$ if $i \in N \backslash D(N, v)$. Then, we get that $\Psi$ satisfies I2ME, IDP, SYM and IMC, but violates EDU.

In view of this fact, we may conclude that the properties used in Theorem 3 are more demanding than the ones used in Theorem 2. Besides, it is necessary to point out that the interval value that satisfies the mentioned properties in Theorem 3 is not unique. Hence, it is still meaningful to further narrow down the category of these indifference values.

\section{The Contracted Interval Banzhaf-like Value and Its Axiomatization}

Since the interval Banzhaf-like value satisfies neither 2ME nor URP, it is still desirable to search for an interval value that is not only median-indifferent to the interval Banzhaf-like value, but also able to accommodate the disadvantages of the interval Banzhaf-like value. With this aim, inspired from Proposition 1 and Theorem 3, we formulate the contracted interval Banzhaf-like value as follows.

Definition 2. The contracted interval Banzhaf-like value is defined as $\mathcal{C B}^{*}: I G^{N} \rightarrow I(\mathbb{R})^{N}$ where $\mathcal{C B}_{i}^{*}(v)=$ $\mathcal{B}_{i}^{*}\left(v_{m}\right) \oplus \frac{1}{2^{|N|-1}} v_{u}(N)$ for any $v \in I G^{N}$ and $i \in N$.

The above notion says that the contracted interval Banzhaf-like value distributes equally payoff uncertainty to players in accordance with the uncertainty of worth for the grand coalition rather than the sum of coalitions' payoff uncertainties.

Reconsider the interval games $v$ in Table 1 and $w$ in Table 2. We have that $\mathcal{C B}^{*}(v)=$ $\left(\left[4, \frac{17}{4}\right],\left[\frac{9}{2}, \frac{19}{4}\right],\left[4, \frac{17}{4}\right]\right)$ and $\mathcal{C B}^{*}(w)=\left(\left[\frac{21}{8}, \frac{27}{8}\right],\left[\frac{37}{8}, \frac{43}{8}\right],\left[\frac{41}{8}, \frac{47}{8}\right]\right)$. It can be verified that $\mathcal{C B}^{*}(v) \sim \mathcal{B}^{*}(v)$ and $\mathcal{C B}^{*}(w) \sim \mathcal{B}^{*}(w)$. 
The next result shows that the contracted interval Banzhaf-like value possesses all the properties mentioned in Theorem 3.

Proposition 2. The contracted interval Banzhaf-like value satisfies I2SA, IDP, SYM, ADD and EDU.

Proof. As $\mathcal{B}^{*}$ satisfies both I2SA and IDP, and $\mathcal{C B}^{*}(v) \sim \mathcal{B}^{*}\left(v_{m}\right)$ for any $v \in I G^{N}$, it can be inferred that $\mathcal{C B}^{*}$ satisfies I2SA and IDP.

Since $\left|\mathcal{C B}_{i}^{*}(v)\right|=\frac{1}{2^{|N|-1}}|v(N)|$ for any $v \in I G^{N}$ and $i \in N$, we obtain that $\mathcal{C B}^{*}$ satisfies EDU.

Let $i, j \in N$ be symmetric players in $v \in I G^{N}$. As $\mathcal{B}^{*}$ satisfies $S Y M$, we get $\mathcal{B}_{i}^{*}\left(v_{m}\right)=\mathcal{B}_{j}^{*}\left(v_{m}\right)$. By EDU, we have $\mathcal{C B}_{i}^{*}(v)=\mathcal{C B}_{j}^{*}(v)$. Thus, $\mathcal{C B}^{*}$ satisfies SYM.

For any $v, w \in I G^{N}$ and $i \in N$, we have $\mathcal{C B}_{i}^{*}(v)=\mathcal{B}_{i}^{*}\left(v_{m}\right) \oplus \frac{1}{2^{|N|-1}} v_{u}(N)$ and $\mathcal{C B}_{i}^{*}(w)=$ $\mathcal{B}_{i}^{*}\left(w_{m}\right) \oplus \frac{1}{2^{|N|-1}} w_{u}(N)$. Then we get

$$
\begin{aligned}
& \mathcal{C B}_{i}^{*}(v) \oplus \mathcal{C B}_{i}^{*}(w)=\mathcal{B}_{i}^{*}\left(v_{m}\right) \oplus \mathcal{B}_{i}^{*}\left(w_{m}\right) \oplus \frac{1}{2^{|N|-1}}\left(v_{u}(N) \oplus w_{u}(N)\right) \\
& \quad=\mathcal{B}_{i}^{*}\left(v_{m}+w_{m}\right) \oplus \frac{1}{2^{|N|-1}}\left(v_{u}+w_{u}\right)(N)=\mathcal{C B}_{i}^{*}(v+w) .
\end{aligned}
$$

Thus, $\mathcal{C B}^{*}$ satisfies ADD.

It can be obtained from Definition 2 that the contracted interval Banzhaf-like value satisfies URP. In contrast, the interval Banzhaf-like value does not possess this property. Moreover, it can be inferred that $\mathcal{C B}^{*}$ satisfies the properties of I2ME and of IMC according to Proposition 2 and Remark 3.

Theorem 4. The contracted interval Banzhaf-like value is the unique interval value satisfying $2 M E, I D P, S Y M$, $A D D$ and $U R P$.

Proof. It can be not difficult verified that $\mathcal{C B}^{*}: I G^{N} \rightarrow I(\mathbb{R})^{N}$ satisfies $2 \mathrm{ME}$, IDP, SYM, ADD and URP.

Let $v \in I G^{N}$ and $\Psi$ be an interval value that satisfies 2ME, IDP, SYM, ADD and URP. It can be verified that $\Psi(v) \sim \mathcal{C B}^{*}(v) \sim \mathcal{B}^{*}\left(v_{m}\right)$. As $v=v_{m}+v_{u}$, it is sufficient to prove that $\Psi\left(v_{m}\right)=\mathcal{C B}^{*}\left(v_{m}\right)$ and $\Psi\left(v_{u}\right)=\mathcal{C B}^{*}\left(v_{u}\right)$.

For any $i \in N, i$ is an uncertainty removing player in $v_{m}$. By URP, we have $\left|\Psi_{i}\left(v_{m}\right)\right|=$ $\left|\mathcal{C B}_{i}^{*}\left(v_{m}\right)\right|=0$. Since $\Psi\left(v_{m}\right) \sim \mathcal{C B}^{*}\left(v_{m}\right)$, it can be inferred that $\Psi_{i}\left(v_{m}\right)=\mathcal{C B}_{i}^{*}\left(v_{m}\right)$ for any $i \in N$. As $v_{u}(S) \sim[0,0]$ for any $S \in 2^{N}$, we can conclude that $i$ is an I-dummy player in $v_{u}$ for any $i \in N$. Thus, $\Psi_{i}\left(v_{u}\right) \sim \mathcal{B}_{i}^{*}\left(v_{u}\right) \sim \mathcal{C B}_{i}^{*}\left(v_{u}\right) \sim[0,0]$.

For any $T \subsetneq N$ and $i \in N \backslash T, i$ is an uncertainty removing player in $z_{T}^{v_{u}(T)}$. By URP, we get $\Psi_{i}\left(z_{T}^{v_{u}(T)}\right)=[0,0]$. Take $i \in T$ and $j \in N \backslash T$. By amalgamating $i$ and $j$, and denoting $p=\{i, j\}$, we obtain $\left(N^{p}, \eta_{T}^{p}\right)$ where $\eta_{T}=z_{T}^{v_{u}(T)}$. We conclude that $\eta_{T}^{p}(S)=[0,0]$ for any $S \subseteq N^{p}$. As $p$ is not only an I-dummy player but also an uncertainty nullifying player in $\eta_{T}^{p}$, we have that $\Psi_{p}\left(\eta_{T}^{p}\right)=[0,0]$. Since $\Psi$ satisfies $2 \mathrm{ME}$, it can be concluded that $\Psi_{i}\left(\eta_{T}\right) \oplus \Psi_{j}\left(\eta_{T}\right)=\Psi_{p}\left(\eta_{T}^{p}\right)$. Thus, $\Psi_{i}\left(\eta_{T}\right)=[0,0]$ for any $i \in T$.

Take $T=N$. Since $\Psi$ satisfies $2 \mathrm{ME}$ and SYM, by the method of induction, it can be inferred that $\Psi_{i}\left(z_{T}^{v_{u}(T)}\right)=\frac{1}{2^{|N|-1}} z_{T}^{v_{u}(T)}(N)=\frac{1}{2^{|N|-1}} v_{u}(N)$ for any $i \in N$. As $v_{u}=\sum_{T \in 2^{N} \backslash\{\varnothing\}} z_{T}^{v_{u}(T)}$, by ADD, we get that

$$
\Psi_{i}\left(v_{u}\right)=\Psi_{i}\left(\sum_{T \in 2^{N} \backslash\{\varnothing\}} z_{T}^{v_{u}(T)}(T)\right)=\sum_{T \in 2^{N} \backslash\{\varnothing\}} \Psi_{i}\left(z_{T}^{v_{u}(T)}(T)\right)=\Psi_{i}\left(z_{N}^{v_{u}(N)}(N)\right)=\frac{1}{2^{|N|-1}} v_{u}(N) .
$$

In order to show the independence between the properties mentioned in Theorem 4, we present the following counterexamples. 


\section{Example 4.}

- $\quad$ Take $\Psi: I G^{N} \rightarrow I(\mathbb{R})^{N}$ where

$$
\Psi_{i}(v)=\sum_{S \subseteq N \backslash\{i\}} \frac{|S| !(|N|-|S|-1) !}{|N| !}\left\{v_{m}(S \cup\{i\}) \ominus v_{m}(S)\right\}
$$

for any $v \in I G^{N}$ and $i \in N$. It can be verified that $\Psi$ satisfies IDP, SYM, ADD and URP, but violates $2 M E$.

- Take $\Psi: I G^{N} \rightarrow I(\mathbb{R})^{N}$ where $\Psi_{i}(v)=\frac{v(N)}{2^{|N|-1}}$ for any $v \in I G^{N}$ and $i \in N$. It can be verified that $\Psi$ satisfies $2 M E, S Y M, A D D$ and URP, but violates IDP.

- Let $\left\{\lambda_{i}\right\}_{i \in N}$ be a set of positive real numbers with $\sum_{i \in N} \lambda_{i}=1$ and $\lambda_{i} \neq \lambda_{j}$ for any $i, j \in N$. Assume that $\lambda_{p}=\lambda_{i}+\lambda_{j}$ for any $i, j \in N$ with $p=\{i, j\}$. Take $\Psi: I G^{N} \rightarrow I(\mathbb{R})^{N}$ as $\Psi_{i}(v)=\mathcal{B}_{i}^{*}\left(v_{m}\right) \oplus \lambda_{i} v_{u}(N)$ for any $v \in I G^{N}$ and $i \in N$. It can be verified that $\Psi$ satisfies $2 M E$, IDP, ADD and URP, but violates SYM.

- Define $\Psi: I G^{N} \rightarrow I(\mathbb{R})^{N}$ as $\Psi_{i}(v)=\mathcal{B}_{i}^{*}\left(v_{m}\right) \oplus \frac{1}{2^{|N|-1}} I^{\circ}$ for any $v \in I G^{N}$ and $i \in N$ where

$$
I^{\circ}=\left\{\begin{array}{cc}
{[-1,1],} & \text { if }\left|v_{u}(N)\right|>0 \\
{[0,0],} & \text { if }\left|v_{u}(N)\right|=0 .
\end{array}\right.
$$

It can be verified that $\Psi$ satisfies $2 M E, I D P, S Y M$ and URP, but violates ADD.

- Define $\Psi: I G^{N} \rightarrow I(\mathbb{R})^{N}$ as $\Psi_{i}(v)=\mathcal{B}_{i}^{*}\left(v_{m}\right) \oplus \frac{1}{2^{|N|-1}} I^{\circ}$ for any $v \in I G^{N}$ and $i \in N$ where $I^{\circ}=$ $\left[-\frac{\overline{v(N)}+v(N)}{2}, \frac{\overline{v(N)}+v(N)}{2}\right]$. It can be verified that $\Psi$ satisfies $2 M E, I D P, S Y M$ and $A D D$, but violates URP.

In view of the above facts, we may conclude that the properties used in Theorem 4 are independent.

\section{Concluding Remarks}

In this paper, we firstly proposed the interval Banzhaf-like value for cooperative interval games to overcome the shortcomings of the interval Banzhaf value introduced in [13]. The interval Banzhaf-like value is well-defined for every possible interval game, whereas the interval Banzhaf value is only definable for size monotonic interval games. As we have shown in Theorem 1, the interval Banzhaf-like value is median-indifferent to the interval Banzhaf value whenever the latter interval value exists. Based on these facts, we conclude that the interval Banzhaf-like value outperforms the interval Banzhaf value in dealing with cooperative games with interval payoffs.

We also introduced two sets of properties about interval values which can be treated as the sufficient conditions for judging whether an interval value is median-indifferent to the interval Banzhaf-like value. In view of the shortcomings of the interval Banzhaf-like value, we came up with the contracted interval Banzhaf-like value by reducing the uncertainty degree of the interval Banzhaf-like value. It has been shown that the contracted interval Banzhaf-like value not just overcomes the mentioned deficiencies of the interval Banzhaf-like value, but also may be characterized by using a set of properties. For these reasons, the theory of the contracted interval Banzhaf-like value may be treated as an improvement of the interval Banzhaf-like value theory.

Recall the definition of interval Shapley-like value in ([14] p. 466), it can be proved that the interval Shapley-like value satisfies the properties of IDP, of SYM, of IMC and of ADD. Besides, it can be verified that the interval Shapley-like value violates 2ME, I2ME, I2SA, EDU and URP (see the counterexample $(N, v)$ in Table 1). Since the interval Shapley-like value, the interval Banzhaf-like value and the contracted interval Banzhaf-like value all can be used as the measures of power in decision making, it is necessary to make a comparison between these interval values. We summarize the commonalities and differences between them in Table 5 and Table 6, respectively. 
Table 5. The commonalities between $\Phi, \mathcal{B}^{*}$ and $\mathcal{C B}^{*}$.

\begin{tabular}{ccccc}
\hline & IDP & SYM & IMC & ADD \\
\hline $\boldsymbol{\Phi}$ & $\mathrm{Y}$ & $\mathrm{Y}$ & $\mathrm{Y}$ & $\mathrm{Y}$ \\
\hline $\mathcal{B}^{*}$ & $\mathrm{Y}$ & $\mathrm{Y}$ & $\mathrm{Y}$ & $\mathrm{Y}$ \\
\hline $\mathcal{C B}^{*}$ & $\mathrm{Y}$ & $\mathrm{Y}$ & $\mathrm{Y}$ & $\mathrm{Y}$ \\
\hline
\end{tabular}

Table 6. The differences between $\Phi, \mathcal{B}^{*}$ and $\mathcal{C B}^{*}$.

\begin{tabular}{ccccccc}
\hline & IEF & 2ME & I2ME & I2SA & EDU & URP \\
\hline $\boldsymbol{\Phi}$ & $\mathrm{Y}$ & $\mathrm{N}$ & $\mathrm{N}$ & $\mathrm{N}$ & $\mathrm{N}$ & $\mathrm{N}$ \\
\hline $\mathcal{B}^{*}$ & $\mathrm{~N}$ & $\mathrm{~N}$ & $\mathrm{Y}$ & $\mathrm{Y}$ & $\mathrm{Y}$ & $\mathrm{N}$ \\
\hline $\mathcal{C B}^{*}$ & $\mathrm{~N}$ & $\mathrm{Y}$ & $\mathrm{Y}$ & $\mathrm{Y}$ & $\mathrm{Y}$ & $\mathrm{Y}$ \\
\hline
\end{tabular}

Based on the results in Tables 5 and 6, we may conclude that the interval Banzhaf-like value and the contracted interval Banzhaf-like value both have more aspects of rationality than the interval Shapley-like value. Moreover, in view of the fairness about the uncertainty distribution among players, the contracted interval Banzhaf-like value performs better than the interval Banzhaf-like value in measuring the power of players.

Author Contributions: Conceptualization, W.F.; Formal analysis, C.T. and W.F.; Funding acquisition, C.T.; Methodology, W.F. and W.H.; Project administration, C.T.; Supervision, C.T. and W.H.; Writing-original draft, W.F.; Writing-review \& editing, W.H. All authors have read and agreed to the published version of the manuscript.

Funding: This work was partly supported by National Natural Science Foundation of China (Nos. 71671188, 71271217, and 71571192), the major project for National NSFC (No. 71790615), Natural Science Foundation of Hunan Province, China (No. 2016JJ1024) and South China Normal University (No.508/671182).

Conflicts of Interest: The authors declare no conflict of interest.

\section{References}

1. Borkotokey, S. Cooperative games with fuzzy coalitions and fuzzy characteristic functions. Fuzzy Sets Syst. 2008, 159, 138-151. [CrossRef]

2. Meng, F.Y.; Zhang, Q. The Shapley function for fuzzy cooperative games with multilinear extension form. Appl. Math. Lett. 2010, 23, 644-650. [CrossRef]

3. Tan, C.; Jiang, Z.; Chen, X.; Ip, W.H. A Banzhaf function for a fuzzy game. IEEE Trans. Fuzzy Syst. 2014, 22, 1489-1502. [CrossRef]

4. Branzei, R.; Branzei, O.; Gök, S.Z.A.; Tijs, S. Cooperative interval games: a survey. Central Eur. J. Oper. Res. 2010, 18, 397-411. [CrossRef]

5. Carpente, L.; Casas-Méndez, B.; García-Jurado, I.; Van Den Nouweland, A. Coalitional interval games for strategic games in which players cooperate. Theory Decis. 2008, 65, 253-269. [CrossRef]

6. Tan, C.; Jiang, Z.; Chen, X. Choquet extension of cooperative games. Asia-Pac. J. Oper. Res. 2013, 30, $229-317$. [CrossRef]

7. Branzei, R.; Dimitrov, D.; Tijs, S. Shapley-like values for interval bankruptcy games. Econ. Bull. 2003, 3, 1-8.

8. Branzei, R.; Mallozzi, L.; Tijs, S. Peer group situations and games with interval uncertainty. Game Theory Appl. 2012, 15, 1-8.

9. Montemanni, R. A Benders decomposition approach for the robust spanning tree problem with interval data. Eur. J. Oper. Res. 2006, 174, 1479-1490. [CrossRef]

10. Moretti, S.; Gök, S.Z.A.; Branzei, R.; Tijs, S. Connection situations under uncertainty and cost monotonic solutions. Comput. Oper. Res. 2011, 38, 1638-1645. [CrossRef]

11. Moore, R.E. Methods and Applications of Interval Analysis; Society for Industrial and Applied Mathematics (SIAM): Philadelphia, PA, USA, 1979; Volume 2.

12. Gök, S.Z.A.; Branzei, R.; Tijs, S. The interval Shapley value: An axiomatization. Central Eur. J. Oper. Res. 2010, $18,131-140$. 
13. Pusillo, L. Banzhaf Like Value for Games with Interval Uncertainty. Czech Econ. Rev. 2013, 7, 5-15.

14. Han, W.; Sun, H.; Xu, G. A new approach of cooperative interval games: The interval core and Shapley value revisited. Oper. Res. Lett. 2012, 40, 462-468. [CrossRef]

15. Shapley, LS. A value for n-person games. Contrib. Theory Games 1953, 2, 307-317.

16. Lehrer, E. An axiomatization of the Banzhaf value. Int. J. Game Theory 1988, 17, 89-99. [CrossRef]

17. Nowak, A.S. On an axiomatization of the Banzhaf value without the additivity axiom. Int. J. Game Theory 1997, 26, 137-141. [CrossRef]

(C) 2020 by the authors. Licensee MDPI, Basel, Switzerland. This article is an open access article distributed under the terms and conditions of the Creative Commons Attribution (CC BY) license (http:/ / creativecommons.org/licenses/by/4.0/). 